\title{
Hiring for Experience: Employer Attitudes Toward Credentials as Proof of Skills Annotated Questionnaire
}

\section{November 2021}

\section{AARP Reskilling Survey Annotated Questionnaire $(n=650)$}

\section{GOALS/QUOTAS:}

n=800 Employers, defined as responsible for their organization's HR functions including screening applicants, recruiting or hiring or conducting and/or supervising training and development programs for employees

- Need to work at company with $100+$ employees

- Soft quotas on industry, company size, and respondent level

- Geography: US

- Qualifications: Be a responsible for HR functions (either hiring or training/development). Sample will target HR personnel when available.

\section{Quota Groups (Not nested):}

The soft quotas are in blue below. Monitoring the target, but expect it will go over/under.

\section{Company Size:}

- Midsized (100-499). S1c=3 or $4(75 \%)$

- Large $(500+)$ : S1c=5 or $6(25 \%)$

Industry: See note at S5 and S6 for quota qualifications

- Agriculture/Mining/Construction 54

- Manufacturing/Transportation/Comm/Electric/Gas/Sanitary Services 161

- Wholesale/Retail 93

- Finance, Insurance, Real Estate, Engineering, Accounting, Management 73

- Hotel, Entertainment Services 97

- Health Services 87

- Legal, Education, Social Services, Museum, Membership 85

- Other 0

- Total 650

Respondent Level:

- Director or higher S6=1-5 (55\%)

- Manager or lower S6=6-7 (45\%) 
PN: Insert start time stamp.

PN: Present "Cell Prompts" (noted below) only if respondent sample is cell phone.

SHOW ALL - INTRODUCTION

Hello, this is calling from SSRS, a national marketing research firm.

[PN: Present if name available] May I please speak to (NAME FROM SAMPLE) or the person responsible for reviewing job applicants at your company?

[PN: Present if no name available] May I please speak to the person responsible for reviewing job applicants at your company?

We are conducting a study about how companies evaluate new job applicants and training programs for current employees and would like to include your, or someone at your company's, opinions.

If you qualify and complete the survey, we would like to offer you a check for $\$ 20$ or the option to donate that money to the American Red Cross as a thank you for your participation.

(IF NECESSARY: Please be assured that no one will contact you to sell you anything as a result of this call. In addition, all of your answers are strictly confidential. Neither your name nor the name of your company will be associated with any of your responses.)

ASK ALL

S1. Are you the senior level executive responsible for making human resources decisions at your organization?

$$
(n=650)
$$

$52 \% \quad$ Yes

$47 \% \quad$ No

1\% (DO NOT READ) Don't know

-- $\quad$ (DO NOT READ) Refused

ASK ALL

S1a. Are you a Manager of Compensation, HR Specialist, OR HR Generalist for your organization? [DO NOT READ RESPONSE SCALE]

$(n=650)$

$\begin{array}{ll}79 \% & \text { Yes } \\ 20 \% & \text { No } \\ <1 \% & \text { (DO NOT READ) Don't know } \\ <1 \% & \text { (DO NOT READ) Refused }\end{array}$


ASK ALL

S1b. Are you responsible for any of the following human resource functions at your organization... [RECORD ANSWER FOR EACH - DO NOT READ RESPONSE SCALE]

a. Screening job applicants, recruiting, or hiring employees [MUST HAVE ITEM A OR C]

$(n=650)$

$95 \%$ Yes

$5 \% \quad$ No

-- $\quad$ (DO NOT READ) Don't know

-- $\quad$ (DO NOT READ) Refused

b. Managing employee benefits and compensation

$(n=650)$

$69 \%$ Yes

$31 \% \quad$ No

-- (DO NOT READ) Don't know

-- $\quad$ (DO NOT READ) Refused

c. Conducting and/or supervising training and development programs for employees [MUST HAVE ITEM A OR C]

$(n=650)$

$68 \%$ Yes

$32 \%$ No

$<1 \%$ (DO NOT READ) Don't know

$-\quad$ (DO NOT READ) Refused

PN: PROCEED TO S1C IF RESPONDENT IS RESPONSIBLE FOR SCREENING AND/OR TRAINING - IF S1B_a=1 OR S1B_c=1, PROCEED. OTHERWISE TERMINATE.

ASK IF S1=1 OR S1A=1 OR (S1B_a=1 OR S1B_c=1)

S1c. And excluding contract or contingent employees, approximately how many employees does your organization have in all locations? [DO NOT READ RESPONSE ITEMS]

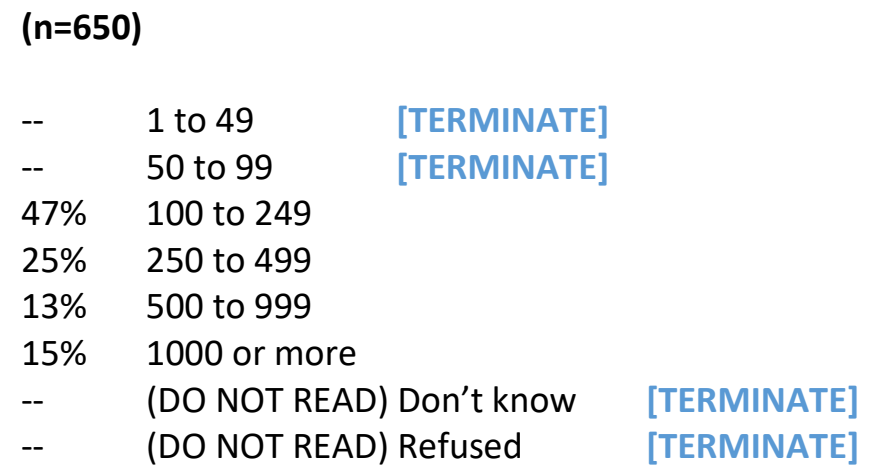


S2. Excluding contract or contingent employees, approximately how many full-time employees does your organization have in all locations? [DO NOT READ RESPONSE ITEMS]

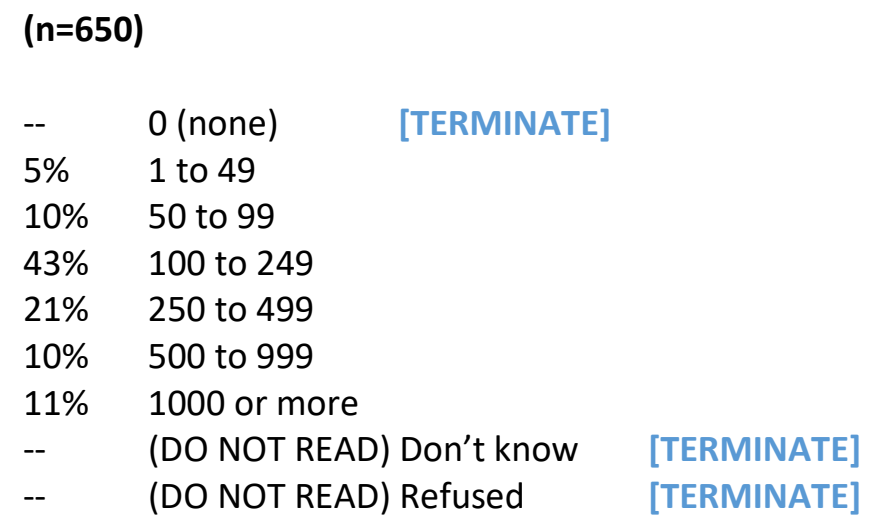

ASK IF S2=1,2,3,4,5,6

S3. Excluding contract or contingent employees, approximately how many part-time employees does your organization have in all locations? [DO NOT READ RESPONSE ITEMS]

$\begin{array}{ll}\text { (n=650) } \\ 19 \% & 0 \text { (none) } \\ 39 \% & 1 \text { to } 49 \\ 13 \% & 50 \text { to } 99 \\ 15 \% & 100 \text { to } 249 \\ 4 \% & 250 \text { to } 499 \\ 3 \% & 500 \text { to } 999 \\ 4 \% & 1000 \text { or more } \\ 3 \% & \text { (DO NOT READ) Don't know } \\ <1 \% & \text { (DO NOT READ) Refused }\end{array}$

PN: IF S1C=3,4,5,6 AND (S2=1,2,3,4,5,6), THEN QUALIFIED ALL OTHERS, TERMINATE.

ASK IF DECISION MAKER AT MIDSIZED/LARGE BUSINESS (S1C=3,4,5,6 and S2=1,2,3,4,5,6)

S4. Which of the following best describes your business? Are you in ... [READ LIST. ENTER ONE ONLY]

$(n=650)$

$\begin{array}{llr}2 \% & \text { Agriculture, Forestry, or Mining } & \text { (PN: Agriculture/Mining/Construction quota) } \\ 6 \% & \text { Construction } & \text { (PN: Agriculture/Mining/Construction quota) } \\ 19 \% & \text { Manufacturing } & \text { (PN: Manufacturing etc. quota) } \\ 6 \% & \text { Transportation, Communication, or Utilities (PN: Manufacturing etc. quota) } \\ 1 \% & \text { Wholesale Trade } & \text { (PN: Wholesale/Retail quota) } \\ 7 \% & \text { Retail Trade (goods, apparel, food service, etc.) (PN: Wholesale/Retail quota) } \\ 6 \% & \text { Finance, Insurance, or Real Estate } & \text { (PN: Finance etc. quota) } \\ <1 \% & \text { Engineering } & \text { (PN: Finance etc. quota) } \\ 1 \% & \text { Accounting, Management } & \text { (PN: Finance etc. quota) } \\ 2 \% & \text { Hotel } & \text { (PN: Hotel etc. quota) } \\ 1 \% & \text { Entertainment Services } & \text { (PN: Hotel etc. quota) }\end{array}$




$\begin{array}{llcl}15 \% & \text { Health Services } & \text { (PN: Health Services etc. quota) } \\ <1 \% & \text { Legal } & \text { (PN: Legall etc. quota) } \\ 10 \% & \text { Education } & \text { (PN: Legall etc. quota) } \\ 5 \% & \text { Social Services, Personal Services, Business Services } & \text { (PN: Legal etc. quota) } \\ <1 \% & \text { Museum, Art Galleries, Amusement Parks } & \text { (PN: Legal etc. quota) } \\ 16 \% & \text { Or something else (SPECIFY) } & \text { (PN: Other) } \\ -- & \text { (DO NOT READ) Don't know } & \\ -- & \text { (DO NOT READ) Refused } & \end{array}$

PN: QUOTA INSTRUCTIONS - IF "OTHER, DON'T KNOW OR REFUSED", QUOTA COMPANY BASED ON SIC CODE IN SAMPLE AS FOLLOWS:

\begin{tabular}{|c|c|c|}
\hline Agriculture, Forestry, or Mining & (PN: Agriculture quota & SIC 1-17) \\
\hline Construction & (PN: Agriculture quota & SIC 1-17) \\
\hline Manufacturing & (PN: Manufacturing quota & SIC 20-49) \\
\hline Transportation, Communication, or & lities (PN: Manufacturing quota & SIC 20-49) \\
\hline Wholesale Trade & (PN: Wholesale/Retail quota & SIC 50-59) \\
\hline \multicolumn{3}{|c|}{ Retail Trade (goods, apparel, food service, etc.) (PN: Wholesale/Retail quota SIC 50-59) } \\
\hline Finance, Insurance, or Real Estate & (PN: Finance etc. quota SIC 6 & $0-69,87-89)$ \\
\hline Engineering & (PN: Finance etc. quota SIC 6 & $0-69,87-89)$ \\
\hline Accounting, Management & (PN: Finance etc. quota & SIC 60-69) \\
\hline Hotel & (PN: Hotel etc. quota & SIC 70-79) \\
\hline Entertainment Services & (PN: Hotel etc. quota & SIC 70-79) \\
\hline Health Services & (PN: Health Services etc. quota & SIC 80) \\
\hline Legal & (PN: Legal etc. quota & SIC 81-86) \\
\hline Education & (PN: Legal etc. quota & SIC 81-86) \\
\hline \multicolumn{3}{|c|}{ Social Services, Personal Services, Business Services $\quad$ (PN: Legal etc. quota SIC 81-86) } \\
\hline Museum, Art Galleries, Amusement & ks $\quad$ (PN: Legal etc. quota & SIC 81-86) \\
\hline
\end{tabular}

PN: Check quotas and allocate respondent to appropriate industry quota group.

ASK IF DECISION MAKER AT MIDSIZED/LARGE BUSINESS (S1C=3,4,5,6 and S2=1,2,3,4,5,6)

S5. In what state is your company located? [READ IF NECESSARY]

[INTERVIEWER INSTRUCTION, IF NEEDED: If company is located in multiple states, in what state do YOU primarily work?]

$(n=650)$

\begin{tabular}{|c|c|}
\hline \multicolumn{2}{|c|}{ Census Region } \\
\hline $16 \%$ & Northeast \\
\hline $27 \%$ & Midwest \\
\hline $35 \%$ & South \\
\hline $22 \%$ & West \\
\hline
\end{tabular}


ASK IF DECISION MAKER AT MIDSIZED/LARGE BUSINESS (S1C=3,4,5,6 and S2=1,2,3,4,5,6)

S6. And what job title is closest to yours? [READ LIST. ENTER ONE ONLY]

$\begin{array}{ll}\text { (n=650) } & \\ 3 \% & \text { C-Level, CEO, CBO, CFO, etc. } \\ 1 \% & \text { Owner } \\ 1 \% & \text { President } \\ 3 \% & \text { Vice President } \\ 21 \% & \text { Director } \\ 28 \% & \text { Manager } \\ 44 \% & \text { Something else } \\ -- & \text { (DO NOT READ) Don't know } \\ -- & \text { (DO NOT READ) Refused }\end{array}$

PN: Check quotas and allocate respondent to Director and above quota group (01-05) or Manager and below quota group (06-07).

ASK IF DECISION MAKER AT MIDSIZED/LARGE BUSINESS (S1C=3,4,5,6 and S2=1,2,3,4,5,6)

S7. And what is your actual job title? [Enter response below]

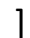

$P N=$ No need to code responses.

PN: ONLY QUALIFIED RESPONDENTS CONTINUE (so "Ask AlI" refers to qualified respondents).

QUALIFICATION:

* ORGANIZATION OF 100 EMPLOYEES OR MORE S1c=3,4,5,6 and $\mathrm{S} 2=1,2,3,4,5,6$ )

$A N D$

*HR DECISION MAKER (S1B_A=1 OR S1B_C=1) 
PN: Insert start time stamp. Present items one at a time.

ASK ALL

1. Many U.S. businesses have been affected by the covid-19 pandemic. Some have experienced a negative impact, some a positive impact, and others experienced no change. In general, which of the following ways has your organization's hiring practices been affected? Have you [RANDOMIZE AND INSERT a-g, ANCHOR h]

a.

changed any of the types of skills you are hiring for?

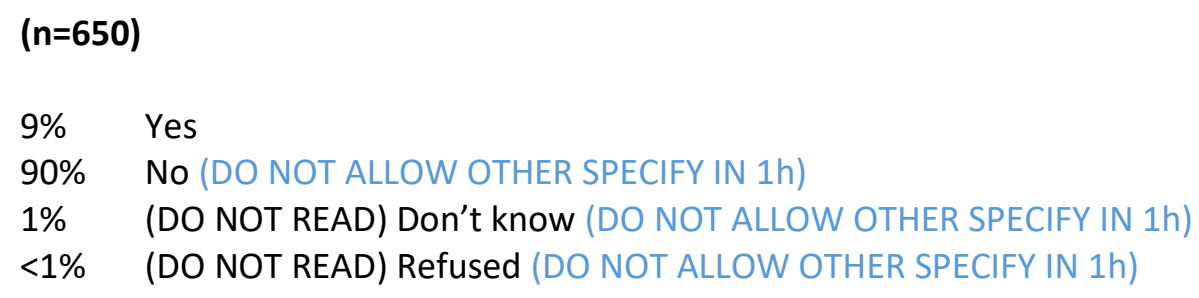

b. lowered the eligibility criteria for any positions?

$(n=650)$

$18 \% \quad$ Yes

$80 \%$ No (DO NOT ALLOW OTHER SPECIFY IN 1h)

$2 \%$ (DO NOT READ) Don't know (DO NOT ALLOW OTHER SPECIFY IN 1h)

$<1 \%$ (DO NOT READ) Refused (DO NOT ALLOW OTHER SPECIFY IN 1h)

c. increased hiring in some departments and decreased it in others?

$(n=650)$

$32 \% \quad$ Yes

$66 \%$ No (DO NOT ALLOW OTHER SPECIFY IN 1h)

2\% (DO NOT READ) Don't know (DO NOT ALLOW OTHER SPECIFY IN 1h)

$1 \%$ (DO NOT READ) Refused (DO NOT ALLOW OTHER SPECIFY IN 1h)

d. increased hiring across the board?

$(n=650)$

$51 \% \quad$ Yes

46\% No (DO NOT ALLOW OTHER SPECIFY IN 1h)

2\% (DO NOT READ) Don't know (DO NOT ALLOW OTHER SPECIFY IN 1h)

$<1 \%$ (DO NOT READ) Refused (DO NOT ALLOW OTHER SPECIFY IN 1h) 
e. decreased hiring across the board?

$(n=650)$

$22 \% \quad$ Yes

76\% No (DO NOT ALLOW OTHER SPECIFY IN 1h)

2\% (DO NOT READ) Don't know (DO NOT ALLOW OTHER SPECIFY IN 1h)

$<1$ (DO NOT READ) Refused (DO NOT ALLOW OTHER SPECIFY IN 1h)

f. focused more on hiring diverse candidates?

$(n=650)$

$51 \% \quad$ Yes

44\% No (DO NOT ALLOW OTHER SPECIFY IN 1h)

$4 \%$ (DO NOT READ) Don't know (DO NOT ALLOW OTHER SPECIFY IN 1h)

$1 \%$ (DO NOT READ) Refused (DO NOT ALLOW OTHER SPECIFY IN 1h)

g. Allowed workers or more workers to telework (could be full time, half-time, in different geographic locations, etc.)

$(n=650)$

$52 \% \quad$ Yes

47\% No (DO NOT ALLOW OTHER SPECIFY IN 1h)

2\% (DO NOT READ) Don't know (DO NOT ALLOW OTHER SPECIFY IN 1h)

$<1 \% \quad$ (DO NOT READ) Refused (DO NOT ALLOW OTHER SPECIFY IN 1h)

h. Has there been some other way your organization's hiring practices have been impacted by the pandemic (specify):

$(n=650)$

$42 \% \quad$ Yes

$52 \% \quad$ No (DO NOT ALLOW OTHER SPECIFY IN 1h)

6\% (DO NOT READ) Don't know (DO NOT ALLOW OTHER SPECIFY IN 1h)

$1 \%$ (DO NOT READ) Refused (DO NOT ALLOW OTHER SPECIFY IN 1h)

ASK ALL

1A. Over the past 12 months, as job applications came in, did your organization struggle to find qualified employees for most open positions, or not?

$(n=650)$

$71 \%$ Yes

$28 \%$ No

1\% (DO NOT READ) Don't know

-- $\quad$ (DO NOT READ) Refused 
ASK ALL

2. In general, when evaluating job applicants, how important are the following skills to your organization for any or most positions? First/next [INSERT ITEM], is this very important, somewhat important, or not important? [RANDOMIZE A-P, ANCHOR Q]

[PN NOTE: 's' and ' $h$ ' designations are for internal use only, not to present in the program]

a. Adaptability to change

$$
(n=650)
$$

$66 \%$ Very important

$29 \%$ Somewhat important

$5 \% \quad$ Not important

$<1 \% \quad$ (DO NOT READ) Don't know

-- (DO NOT READ) Refused

b. Basic skills, like math, basic writing, or reading skills

$$
(n=650)
$$

$68 \%$ Very important

$27 \%$ Somewhat important

$5 \% \quad$ Not important

1\% (DO NOT READ) Don't know

-- $\quad$ (DO NOT READ) Refused

c. Technical skills like engineering, mechanical, machine operation, etc.

$(n=650)$

29\% Very important

$25 \%$ Somewhat important

$44 \% \quad$ Not important

2\% (DO NOT READ) Don't know

$<1 \% \quad$ (DO NOT READ) Refused

e. Management or leadership skills

$(n=650)$

$\begin{array}{ll}34 \% & \text { Very important } \\ 47 \% & \text { Somewhat important } \\ 17 \% & \text { Not important } \\ 2 \% & \text { (DO NOT READ) Don't know } \\ <1 \% & \text { (DO NOT READ) Refused }\end{array}$


f. Team work or collaboration

$(n=650)$

83\% Very important

$15 \%$ Somewhat important

2\% Not important

-- $\quad$ (DO NOT READ) Don't know

-- $\quad$ (DO NOT READ) Refused

g. Information and digital technology

$(n=650)$

27\% Very important

$43 \%$ Somewhat important

$29 \% \quad$ Not important

$1 \%$ (DO NOT READ) Don't know

$<1 \% \quad$ (DO NOT READ) Refused

h. Analytical skills like collecting and analyzing data for decision making

$(n=650)$

29\% Very important

$39 \%$ Somewhat important

$30 \% \quad$ Not important

2\% (DO NOT READ) Don't know

$<1 \%$ (DO NOT READ) Refused

i. Fluency in another language other than English

$(n=650)$

7\% Very important

$33 \%$ Somewhat important

$59 \% \quad$ Not important

$<1 \%$ (DO NOT READ) Don't know

$1 \%$ (DO NOT READ) Refused

j. Customer service skills

$(n=650)$

68\% Very important

19\% Somewhat important

$13 \% \quad$ Not important

$<1 \%$ (DO NOT READ) Don't know

$<1 \% \quad$ (DO NOT READ) Refused 
k. Creativity and innovation

$$
(n=650)
$$

$20 \%$ Very important

$47 \%$ Somewhat important

$32 \% \quad$ Not important

$1 \%$ (DO NOT READ) Don't know

$<1 \% \quad$ (DO NOT READ) Refused

I. Problem solving skills

$$
(n=650)
$$

66\% Very important

$28 \%$ Somewhat important

$5 \% \quad$ Not important

1\% (DO NOT READ) Don't know

-- (DO NOT READ) Refused

m. Mentoring, coaching, training skills

$$
(n=650)
$$

29\% Very important

51\% Somewhat important

$17 \% \quad$ Not important

2\% (DO NOT READ) Don't know

$<1 \% \quad$ (DO NOT READ) Refused

n. Artistic or design skills

$$
(n=650)
$$

7\% Very important

$24 \%$ Somewhat important

$68 \% \quad$ Not important

1\% (DO NOT READ) Don't know

$<1 \% \quad$ (DO NOT READ) Refused

o. Work ethic and professionalism

$$
(n=650)
$$

$\begin{array}{ll}90 \% & \text { Very important } \\ 8 \% & \text { Somewhat important } \\ 1 \% & \text { Not important } \\ <1 \% & \text { (DO NOT READ) Don't know } \\ -- & \text { (DO NOT READ) Refused }\end{array}$


p. Presentation and public speaking skills

$(n=650)$

$15 \%$ Very important

$36 \%$ Somewhat important

$48 \% \quad$ Not important

1\% (DO NOT READ) Don't know

$<1 \% \quad$ (DO NOT READ) Refused

q. $\quad$ Are there any other skills that are very or somewhat important when evaluating job applicants? (If Yes, say, What others, and are they very important or somewhat important?: (specify) If No, code as "not important" (DO NOT ALLOW OTHER SPECIFY IF NOT IMPORTANT/DK/REF)).

$(n=650)$

$23 \% \quad$ Very important

$2 \%$ Somewhat important

$55 \% \quad$ Not important

$18 \%$ (DO NOT READ) Don't know

2\% (DO NOT READ) Refused

PN: ROTATE WHICH MES FIRST, ALL OR NO POSITIONS. ROTATE RESPONSE OPTIONS IN SAME ORDER AS TEXT IN QUESTION

ASK ALL

3. As I read each of the following types of degrees or certifications, please indicate whether your organization currently requires it for [ROTATE SCALE: all, most, some, or no job applicant positions / none, some, most, or all job applicant positions]? First/next, a [INSERT ITEM --KEEP A/B/C/D TOGETHER AND E/F TOGETHER, ROTATE THE TWO GROUPS]? [REPEAT SCALE THE FIRST TIME AND THEN ONLY AS NEEDED.]

a. High school diploma or G-E-D

$(n=650)$

$\begin{array}{ll}12 \% & \text { No position/none } \\ 20 \% & \text { Some } \\ 15 \% & \text { Most } \\ 52 \% & \text { All } \\ 1 \% & \text { (DO NOT READ) Don't know } \\ -- & \text { (DO NOT READ) Refused }\end{array}$


b. Associates degree, or 2-year college degree

$\begin{array}{ll}\text { (n=650) } & \\ & \\ 24 \% & \text { No position/none } \\ 64 \% & \text { Some } \\ 9 \% & \text { Most } \\ 2 \% & \text { All } \\ -- & \text { (DO NOT READ) Don't know } \\ -- & \text { (DO NOT READ) Refused }\end{array}$

c. Bachelor's degree, or 4-year college degree

$\begin{array}{ll}\text { (n=650) } & \\ 24 \% & \text { No position/none } \\ 64 \% & \text { Some } \\ 10 \% & \text { Most } \\ 2 \% & \text { All } \\ -- & \text { (DO NOT READ) Don't know } \\ -- & \text { (DO NOT READ) Refused }\end{array}$

d. Advanced degree, such as Masters, Ph.D, law degree, et cetera

$\begin{array}{ll}\text { (n=650) } & \\ & \\ 55 \% & \text { No position/none } \\ 42 \% & \text { Some } \\ 2 \% & \text { Most } \\ 1 \% & \text { All } \\ <1 \% & \text { (DO NOT READ) Don't know } \\ -- & \text { (DO NOT READ) Refused }\end{array}$

e. Completion of a certification program

$\begin{array}{ll}\text { (n=650) } & \\ & \\ 23 \% & \text { No position/none } \\ 53 \% & \text { Some } \\ 16 \% & \text { Most } \\ 7 \% & \text { All } \\ <1 \% & \text { (DO NOT READ) Don't know } \\ <1 \% & \text { (DO NOT READ) Refused }\end{array}$


f. Completion of specific credentials for specific skills

$\begin{array}{ll}\text { (n=650) } & \\ & \\ 19 \% & \text { No position/none } \\ 55 \% & \text { Some } \\ 18 \% & \text { Most } \\ 7 \% & \text { All } \\ <1 \% & \text { (DO NOT READ) Don't know } \\ -- & \text { (DO NOT READ) Refused }\end{array}$

ASK ALL

4. Has your organization ever hired any workers who lack the educational degrees that may be typically required for a job but their experience or other training made them well suited for the job? [IF YES, ASK:

Would that be often or occasionally?]

$\begin{array}{ll}\text { (n=650) } & \\ 12 \% & \text { Yes, often } \\ 62 \% & \text { Yes, occasionally } \\ 21 \% & \text { No } \\ 5 \% & \text { (DO NOT READ) Don't know } \\ <1 \% & \text { (DO NOT READ) Refused }\end{array}$

\section{PN: ROTATE RESPONSE OPTIONS IN SAME ORDER AS QUESTION TEXT}

\section{ASK ALL}

5. Thinking about your current talent acquisition practices, would you say that your organization leans toward (screening for education attainment first and skills second, or the other way around - skills first, then educational attainment/skills first, then educational attainment, or the other way around - screening for education attainment first and skills second)?

$(n=650)$

$19 \%$ Screen for educational attainment first, skills second

76\% Screen for skills first, educational attainment second

4\% (DO NOT READ) Don't know

$1 \%$ (DO NOT READ) Refused 
ASK ALL

6. When evaluating job applicants today as compared to about 5 years ago, would you say the value your organization places on educational attainment alongside other job skills has (increased, decreased / decreased, increased) or stayed the same?

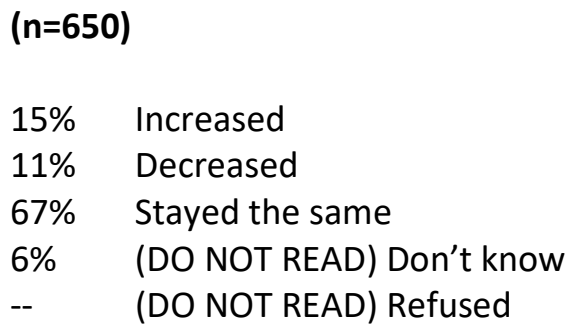

PN: ROTATE WHICH COMES FIRST, AGREE OR DISAGREE. SAME ROTATION THROUGHOUT QUESTION.

\section{ASK ALL}

7. Now tell me if you (agree) or (disagree) with each of the following statements [RANDOMIZE a-e]... And do you strongly or somewhat (AGREE/DISAGREE)?

a. An educational degree is a reliable representation of a candidate's skills and knowledge

$(n=650)$

$13 \%$ Strongly agree

$53 \%$ Somewhat agree

$4 \%$ (DO NOT READ) Neither agree nor disagree

$20 \%$ Somewhat disagree

$10 \%$ Strongly disagree

$<1 \% \quad$ (DO NOT READ) Don't know

$<1 \% \quad$ (DO NOT READ) Refused

b. Work and life experience are a reliable representation of a candidate's skills and knowledge $(n=650)$

$\begin{array}{ll}38 \% & \text { Strongly agree } \\ 53 \% & \text { Somewhat agree } \\ 1 \% & \text { (DO NOT READ) Neither agree nor disagree } \\ 7 \% & \text { Somewhat disagree } \\ 1 \% & \text { Strongly disagree } \\ <1 \% & \text { (DO NOT READ) Don't know } \\ -- & \text { (DO NOT READ) Refused }\end{array}$


c. Work and life experience can substitute for a lack of required degree

$$
(n=650)
$$

$34 \%$ Strongly agree

$48 \%$ Somewhat agree

$2 \% \quad$ (DO NOT READ) Neither agree nor disagree

$11 \%$ Somewhat disagree

$5 \% \quad$ Strongly disagree

1\% (DO NOT READ) Don't know

$<1 \% \quad$ (DO NOT READ) Refused

d. A relevant credential or certification can substitute for a lack of required degree

$(n=650)$

25\% Strongly agree

$58 \%$ Somewhat agree

2\% (DO NOT READ) Neither agree nor disagree

9\% Somewhat disagree

$5 \%$ Strongly disagree

1\% (DO NOT READ) Don't know

$<1 \% \quad$ (DO NOT READ) Refused

e. Pre-employment skills or ability tests are a better indication of skills than an educational degree or credential attainment.

$(n=650)$

18\% Strongly agree

$48 \%$ Somewhat agree

6\% (DO NOT READ) Neither agree nor disagree

$23 \%$ Somewhat disagree

4\% Strongly disagree

2\% (DO NOT READ) Don't know

$<1 \% \quad$ (DO NOT READ) Refused

PN: ROTATE WHICH COMES FIRST, EASY OR DIFFICULT. SAME ROTATION THROUGHOUT QUESTION. 


\section{ASK ALL}

8. When your organization considers the skills they believe are needed to stay competitive and successful in the future, how [easy or difficult / difficult or easy] is it for you to predict the need for each of the specific skills: [RANDOMIZE AND INSERT a-e]

ASK/REPEAT SCALE ONLY FOR FIRST ITEM AND THEN 'AS NEEDED'] Is this a skill that is [easy or difficult /difficult or easy] to predict needing when planning for future jobs? And is that very or somewhat (easy/difficult)?]

a. Digital skills such as digital marketing and web design

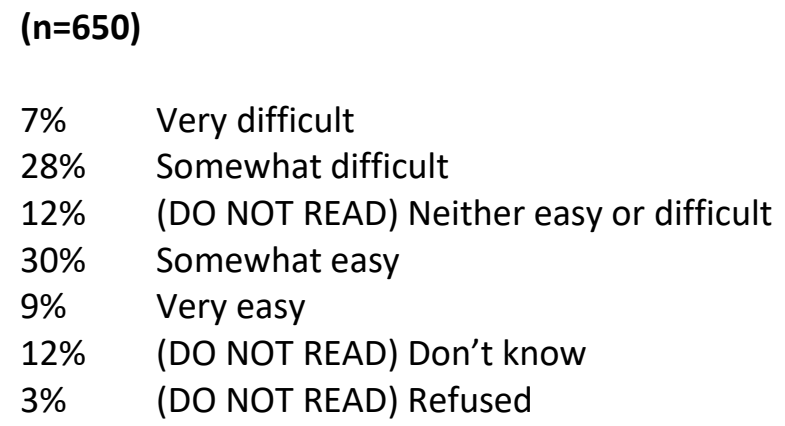

b. Hard technical skills such as cloud computing and machine learning

$(n=650)$

$\begin{array}{ll}11 \% & \text { Very difficult } \\ 33 \% & \text { Somewhat difficult } \\ 7 \% & \text { (DO NOT READ) Neither easy or difficult } \\ 26 \% & \text { Somewhat easy } \\ 12 \% & \text { Very easy } \\ 10 \% & \text { (DO NOT READ) Don't know } \\ 2 \% & \text { (DO NOT READ) Refused }\end{array}$

c. Industry specific skills relevant to your industry

$(n=650)$

4\% Very difficult

$21 \%$ Somewhat difficult

3\% (DO NOT READ) Neither easy or difficult

$41 \%$ Somewhat easy

$26 \%$ Very easy

4\% (DO NOT READ) Don't know

$1 \% \quad$ (DO NOT READ) Refused 
d. Emerging hybrid skills such as data science and experience design

$$
(n=650)
$$

$11 \% \quad$ Very difficult

$37 \%$ Somewhat difficult

$14 \%$ (DO NOT READ) Neither easy or difficult

$16 \%$ Somewhat easy

7\% Very easy

12\% (DO NOT READ) Don't know

3\% (DO NOT READ) Refused

e. Soft skills such as creativity and leadership

$$
\begin{array}{ll}
\text { (n=650) } & \\
5 \% & \text { Very difficult } \\
28 \% & \text { Somewhat difficult } \\
5 \% & \text { (DO NOT READ) Neither easy or difficult } \\
39 \% & \text { Somewhat easy } \\
18 \% & \text { Very easy } \\
3 \% & \text { (DO NOT READ) Don't know } \\
1 \% & \text { (DO NOT READ) Refused }
\end{array}
$$

\section{PN: ROTATE WHICH COMES FIRST, EASY OR DIFFICULT. SAME ROTATION THROUGHOUT QUESTION.}

\section{ASK ALL}

9. Thinking again of when your organization forecasts for future jobs and the skills needed for those jobs, how (easy or difficult/difficult or easy) is it for you and your organization to: [RANDOMIZE AND INSERT a-d]

ASK/REPEAT ONLY FOR FIRST ITEM AND THEN 'AS NEEDED': Is this (easy or difficult/difficult or easy) for your organization to do when planning for future jobs and skills needed? And is that very or somewhat [easy/difficult?]

a. create job descriptions that accurately reflect the specific skills needed for the job?

$\begin{array}{ll}\text { (n=650) } & \\ 1 \% & \text { Very difficult } \\ 12 \% & \text { Somewhat difficult } \\ 1 \% & \text { (DO NOT READ) Neither easy or difficult } \\ 41 \% & \text { Somewhat easy } \\ 44 \% & \text { Very easy } \\ 1 \% & \text { (DO NOT READ) Don't know } \\ -- & \text { (DO NOT READ) Refused }\end{array}$


b. clearly define the difference between a competency and a skill?

$\begin{array}{ll}\text { ( } n=650) & \\ 3 \% & \text { Very difficult } \\ 23 \% & \text { Somewhat difficult } \\ 3 \% & \text { (DO NOT READ) Neither easy or difficult } \\ 44 \% & \text { Somewhat easy } \\ 24 \% & \text { Very easy } \\ 3 \% & \text { (DO NOT READ) Don't know } \\ -- & \text { (DO NOT READ) Refused }\end{array}$

c. implement or update the technology (like applicant tracking) needed to capture new types of skills?

$\begin{array}{ll}\text { (n=650) } & \\ 5 \% & \text { Very difficult } \\ 25 \% & \text { Somewhat difficult } \\ 4 \% & \text { (DO NOT READ) Neither easy or difficult } \\ 42 \% & \text { Somewhat easy } \\ 22 \% & \text { Very easy } \\ 3 \% & \text { (DO NOT READ) Don't know } \\ 1 \% & \text { (DO NOT READ) Refused }\end{array}$

d. train and orient HR and other leadership on how to best assess job candidates for emerging and future skills?

$(n=650)$

$4 \% \quad$ Very difficult

$24 \%$ Somewhat difficult

$3 \% \quad$ (DO NOT READ) Neither easy or difficult

$43 \%$ Somewhat easy

$24 \%$ Very easy

3\% (DO NOT READ) Don't know

$<1 \% \quad$ (DO NOT READ) Refused 
PN: ROTATE WHICH COMES FIRST FOR EVERY OTHER RESPONDENT, VERY IMPORTANT OR NOT AT ALL IMPORTANT. SAME ROTATION THROUGHOUT QUESTION.

ASK ALL

10. And now please tell me how important each of the following are to your organization when evaluating a job applicant's skills: [RANDOMIZE a-e INSERT]... would you say this is a very important consideration, somewhat important, or not important?

a. Length of time working and/or applying specific skill or skills

$$
\begin{array}{ll}
\text { (n=650) } & \\
51 \% & \text { Very important } \\
44 \% & \text { Somewhat important } \\
5 \% & \text { Not important } \\
1 \% & \text { (DO NOT READ) Don't know } \\
<1 \% & \text { (DO NOT READ) Refused }
\end{array}
$$

b. Certifications or credentials in a skill or skills

$$
(n=650)
$$

$\begin{array}{ll}38 \% & \text { Very important } \\ 52 \% & \text { Somewhat important } \\ 9 \% & \text { Not important } \\ <1 \% & \text { (DO NOT READ) Don't know } \\ <1 \% & \text { (DO NOT READ) Refused }\end{array}$

c. Experience and use of the skill or skills in previous positions

$$
\begin{array}{ll}
\text { (n=650) } & \\
56 \% & \text { Very important } \\
41 \% & \text { Somewhat important } \\
3 \% & \text { Not important } \\
1 \% & \text { (DO NOT READ) Don't know } \\
<1 \% & \text { (DO NOT READ) Refused }
\end{array}
$$

d. Successful completion of pre-employment skills tests

$$
(n=650)
$$

$\begin{array}{ll}31 \% & \text { Very important } \\ 36 \% & \text { Somewhat important } \\ 30 \% & \text { Not important } \\ 2 \% & \text { (DO NOT READ) Don't know } \\ 1 \% & \text { (DO NOT READ) Refused }\end{array}$


e. The reputation of the institution or organization that offers skills training

$\begin{array}{ll}\text { (n=650) } & \\ 35 \% & \text { Very important } \\ 45 \% & \text { Somewhat important } \\ 18 \% & \text { Not important } \\ 1 \% & \text { (DO NOT READ) Don't know } \\ <1 \% & \text { (DO NOT READ) Refused }\end{array}$

ASK ALL

11. Does your organization use any applicant tracking software to assist with finding, screening, matching, and hiring job candidates?

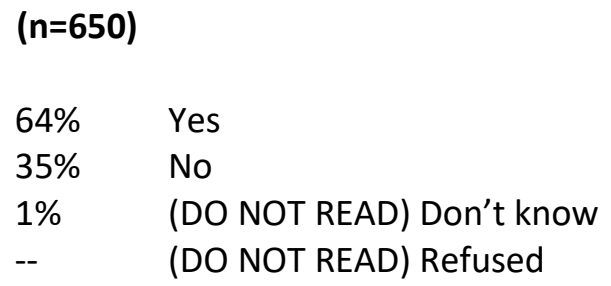

ASK ALL

12. Now I'm going to read some challenges organizations may face when checking a job applicants' credentials as proof of skills required for a certain position. After each, please tell me if your organization is currently faced with that challenge. First/next [INSERT ITEM. RANDOMIZE a- g]? [IF NEEDED: Is your organization currently faced with this challenge, or not?]

a. It is difficult or impossible to find a valid source to confirm credentials, such as an institution, training center, instructor or online program

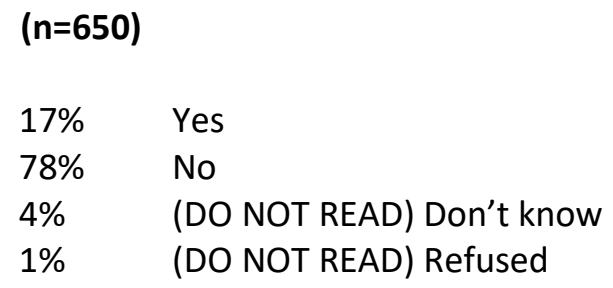

b. Not enough time to thoroughly assess each credential

$(n=650)$

$\begin{array}{ll}25 \% & \text { Yes } \\ 72 \% & \text { No } \\ 2 \% & \text { (DO NOT READ) Don't know } \\ <1 \% & \text { (DO NOT READ) Refused }\end{array}$


c. Not enough trained staff to thoroughly assess each credential

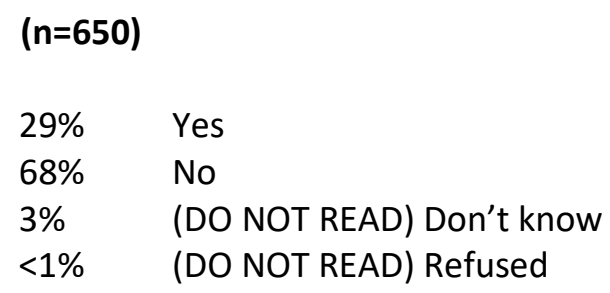

d. Hiring managers or other organizational leaders are distrusting of the validity of applicant credentials

$(n=650)$

$\begin{array}{ll}19 \% & \text { Yes } \\ 79 \% & \text { No } \\ 2 \% & \text { (DO NOT READ) Don't know } \\ <1 \% & \text { (DO NOT READ) Refused }\end{array}$

e. The current applicant tracking process or other related software is not configured to identify and confirm all the possible kinds of credentials

$(n=650)$

$\begin{array}{ll}28 \% & \text { Yes } \\ 65 \% & \text { No } \\ 6 \% & \text { (DO NOT READ) Don't know } \\ 1 \% & \text { (DO NOT READ) Refused }\end{array}$

f. The credentials aren't clear about specific skills gained or enhanced

$(n=650)$

$28 \% \quad$ Yes

$69 \% \quad$ No

3\% (DO NOT READ) Don't know

$<1 \% \quad$ (DO NOT READ) Refused

g. Past experience of hiring without validating credentials had a negative impact on a department or the organization

$(n=650)$

$30 \% \quad$ Yes

$65 \%$ No

4\% (DO NOT READ) Don't know

$<1 \% \quad$ (DO NOT READ) Refused 
AT ALL. SAME ROTATION THROUGHOUT QUESTION.

ASK ALL

13. To what extent are each of the following a (major barrier), a (minor barrier), or not a barrier at all to accepting credentials as proof of a job applicant's skills. First/next [INSERT RANDOMIZE a- e]? [IF NEEDED: Is this a (major barrier), a (minor barrier), or not a barrier at all?]

a. Difficulty determining the validity of a credential

$$
\begin{array}{ll}
(\mathbf{n = 6 5 0 )}) & \\
6 \% & \text { Major barrier } \\
32 \% & \text { Minor barrier } \\
59 \% & \text { Not a barrier at all } \\
3 \% & \text { (DO NOT READ) Don't know } \\
<1 \% & \text { (DO NOT READ) Refused }
\end{array}
$$

b. Existing hiring processes don't include credentials in evaluating candidates

$(n=650)$

$\begin{array}{ll}8 \% & \text { Major barrier } \\ 28 \% & \text { Minor barrier } \\ 60 \% & \text { Not a barrier at all } \\ 4 \% & \text { (DO NOT READ) Don't know } \\ <1 \% & \text { (DO NOT READ) Refused }\end{array}$

c. Overall organizational bias for degrees remains

$(n=650)$

5\% Major barrier

$30 \% \quad$ Minor barrier

$61 \% \quad$ Not a barrier at all

$4 \%$ (DO NOT READ) Don't know

1\% (DO NOT READ) Refused

d. Organizational leadership bias for degrees remains

$(n=650)$

$\begin{array}{ll}5 \% & \text { Major barrier } \\ 30 \% & \text { Minor barrier } \\ 61 \% & \text { Not a barrier at all } \\ 3 \% & \text { (DO NOT READ) Don't know } \\ <1 \% & \text { (DO NOT READ) Refused }\end{array}$


e. Infrastructure and technology related to searching for and identifying candidates
$(n=650)$
9\% Major barrier
32\% Minor barrier
$55 \% \quad$ Not a barrier at all
3\% (DO NOT READ) Don't know
$<1 \% \quad$ (DO NOT READ) Refused

PN: ROTATE WHICH COMES FIRST FOR EVERY OTHER RESPONDENT, VERY HELPFUL OR NOT AT ALL HELPFUL. SAME ROTATION THROUGHOUT QUESTION.

\section{ASK IF Q13=1-2 TO ANY}

14. To help overcome those barriers, please tell me how helpful each of the following would be to your organization [RANDOMIZE and INSERT a-e]

AFTER FIRST ITEM ONLY, READ: Would that be very helpful, somewhat helpful, or not at all helpful? [THEN USE FOR PROMPT IF NEEDED]

a. A trusted third-party solution, updated regularly, that brings transparency to the credentialing marketplace.

$$
\begin{array}{ll}
\text { (n=419) } & \\
24 \% & \text { Very helpful } \\
46 \% & \text { Somewhat helpful } \\
30 \% & \text { Not at all helpful } \\
1 \% & \text { (DO NOT READ) Don't know } \\
-- & \text { (DO NOT READ) Refused }
\end{array}
$$

b. A trusted third-party solution that integrates all possible skills and educational credentials INTO application tracking systems

$(n=419)$

$\begin{array}{ll}32 \% & \text { Very helpful } \\ 44 \% & \text { Somewhat helpful } \\ 24 \% & \text { Not at all helpful } \\ <1 \% & \text { (DO NOT READ) Don't know } \\ -- & \text { (DO NOT READ) Refused }\end{array}$


c. Learning if and how other organizations are accepting certain credentials as proof of skills

$$
(n=419)
$$

$21 \% \quad$ Very helpful

$52 \%$ Somewhat helpful

$26 \% \quad$ Not at all helpful

$<1 \%$ (DO NOT READ) Don't know

-- $\quad$ (DO NOT READ) Refused

d. Skills and credentialing training for all HR leaders, managers, staff

$$
(n=419)
$$

$\begin{array}{ll}39 \% & \text { Very helpful } \\ 43 \% & \text { Somewhat helpful } \\ 17 \% & \text { Not at all helpful } \\ <1 \% & \text { (DO NOT READ) Don't know } \\ -- & \text { (DO NOT READ) Refused }\end{array}$

e. $\quad$ Funding for technology or organizational culture change initiatives

$$
(n=419)
$$

$\begin{array}{ll}33 \% & \text { Very helpful } \\ 40 \% & \text { Somewhat helpful } \\ 25 \% & \text { Not at all helpful } \\ 1 \% & \text { (DO NOT READ) Don't know } \\ <1 \% & \text { (DO NOT READ) Refused }\end{array}$

ASK ALL

15. Thinking about all the possible kinds of non-conventional skills and credentials emerging these days (such as creativity, innovation, and adaptability), what one factor would lead you to trust and value a credential you haven't encountered before? (PROBE AS NEEDED FOR CLARITY)

[OPEN END] 
READ TO ALL: Now l'd like to ask a few questions about the training and development opportunities your organization offers your existing employees.

ASK ALL

16. Does your organization offer the following to any employees in your organization? [RANDOMIZE and READ a-c]

a. Organizational training around policies, benefits, structure, etc.

$\begin{array}{ll}\text { (n=650) } & \\ 92 \% & \text { Yes } \\ 8 \% & \text { No } \\ 1 \% & \text { (DO NOT READ) Don't know } \\ -- & \text { (DO NOT READ) Refused }\end{array}$

b. Operational/On the Job training such as around internal processes, equipment, or customer service.

$(n=650)$

$\begin{array}{ll}94 \% & \text { Yes } \\ 6 \% & \text { No } \\ <1 \% & \text { (DO NOT READ) Don't know } \\ -- & \text { (DO NOT READ) Refused }\end{array}$

c. Compliance training such as diversity \& inclusion, occupational safety, ethics, or data protection. $(n=650)$

$92 \% \quad$ Yes

$7 \% \quad$ No

1\% (DO NOT READ) Don't know

-- $\quad$ (DO NOT READ) Refused

PN: ROTATE WHICH COMES FIRST, ALL OR NO POSITIONS. ROTATE RESPONSE OPTIONS IN SAME ORDER AS TEXT IN QUESTION 


\section{ASK ALL}

17. And which of the following types of skills training, that are not part of a tuition reimbursement program, does your organization offer to [all, some, or none / none, some, or all] of their employees: [RANDOMIZE and READ a-c]

ASK/PROMPT AFTER FIRST ITEM ONLY 'And is that for [all, some, or no employees/ none, some, or all employees] in your organization?' THEN USE AS A PROMPT AS NEEDED]

a. Communication skills such as writing, public speaking, negotiating, conflict resolution

$\begin{array}{ll}\text { (n=650) } & \\ 36 \% & \text { No employees } \\ 40 \% & \text { Some employees } \\ 22 \% & \text { All employees } \\ 2 \% & \text { (DO NOT READ) Don't know } \\ <1 \% & \text { (DO NOT READ) Refused }\end{array}$

b. Digital skills such as using different devices, applications, networks to access/manage information $(n=650)$

$\begin{array}{ll}28 \% & \text { No employees } \\ 46 \% & \text { Some employees } \\ 24 \% & \text { All employees } \\ 2 \% & \text { (DO NOT READ) Don't know } \\ <1 \% & \text { (DO NOT READ) Refused }\end{array}$

c. Technical or mechanical skills such as finance or accounting, operating machinery, web development or programming languages

$(n=650)$

$\begin{array}{ll}32 \% & \text { No employees } \\ 49 \% & \text { Some employees } \\ 17 \% & \text { All employees } \\ 2 \% & \text { (DO NOT READ) Don't know } \\ <1 \% & \text { (DO NOT READ) Refused }\end{array}$

d. Leadership skills such as people or department management or project coordination, coaching, instruction

$(n=650)$

$17 \% \quad$ No employees

$59 \%$ Some employees

$22 \% \quad$ All employees

1\% (DO NOT READ) Don't know

$<1 \% \quad$ (DO NOT READ) Refused 


\section{ASK ALL}

18. Which of the following statements, if any, best represents your organization's approach to providing skill learning opportunities to employees - also known as "re-skilling" or "up-skilling"? Would you say....(READ LIST)

\section{$(n=650)$}

20\% You offer more than the competition in terms of re-skilling or up-skilling your employees

$61 \%$ You are mostly in line with the competition but could improve

$12 \% \quad$ You are not in line with the competition in many areas of re-skilling or up-skilling employees

$3 \% \quad$ (DO NOT READ) None of these reflect my view

4\% (DO NOT READ) Don't know

$<1 \% \quad$ (DO NOT READ) Refused

\section{ASK ALL}

19. Organizations face many barriers to providing employees the training they need to enhance current skills and learn new ones. After each possible barrier I read, let me know if it is one your organization is currently facing. First/next [INSERT ITEM; RANDOMIZE a-f] . [IF NEEDED: Is this a barrier your organization is currently facing, or not?] [ANCHOR g. something else]

a. Not enough funds

$(n=650)$

$$
\begin{array}{ll}
27 \% & \text { Yes } \\
70 \% & \text { No } \\
2 \% & \text { (DO NOT READ) Don't know } \\
<1 \% & \text { (DO NOT READ) Refused }
\end{array}
$$

b. How to measure the ROI (or return on investment) of training

$(n=650)$

$$
\begin{array}{ll}
30 \% & \text { Yes } \\
65 \% & \text { No } \\
5 \% & \text { (DO NOT READ) Don't know } \\
<1 \% & \text { (DO NOT READ) Refused }
\end{array}
$$

c. Lack of leadership support

$(n=650)$

$$
\begin{array}{ll}
21 \% & \text { Yes } \\
77 \% & \text { No } \\
2 \% & \text { (DO NOT READ) Don't know } \\
<1 \% & \text { (DO NOT READ) Refused }
\end{array}
$$


d. Uncertainty about the legitimacy or application of newer skills training

$(n=650)$
$16 \%$ Yes
$81 \%$ No
2\% (DO NOT READ) Don't know
$<1 \% \quad$ (DO NOT READ) Refused

e. Uncertainty within the organization about the skills needed among current employees

$(n=650)$

$\begin{array}{ll}17 \% & \text { Yes } \\ 80 \% & \text { No } \\ 3 \% & \text { (DO NOT READ) Don't know } \\ <1 \% & \text { (DO NOT READ) Refused }\end{array}$

f. Minimal or decreased employee enrollment in training year after year

$(n=650)$

$\begin{array}{ll}23 \% & \text { Yes } \\ 74 \% & \text { No } \\ 3 \% & \text { (DO NOT READ) Don't know } \\ <1 \% & \text { (DO NOT READ) Refused }\end{array}$

g. Something else - can you specify: (IF NO, DK, REF, DO NOT ALLOW TEXT)

$(n=650)$

$\begin{array}{ll}7 \% & \text { Yes } \\ 82 \% & \text { No } \\ 10 \% & \text { (DO NOT READ) Don't know } \\ 1 \% & \text { (DO NOT READ) Refused }\end{array}$

PN: ROTATE INCREASE, DECREASE, REMAIN STABLE. ROTATE RESPONSE OPTIONS IN SAME ORDER AS TEXT IN QUESTION 
ASK ALL

20. Looking ahead over the next 3 to 5 years, do you expect your learning and development budget to [increase], [decrease], or [remain stable]?

$\begin{array}{ll}\text { (n=650) } & \\ 43 \% & \text { Increase } \\ 2 \% & \text { Decrease } \\ 51 \% & \text { Remain stable } \\ 3 \% & \text { (DO NOT READ) Don't know } \\ <1 \% & \text { (DO NOT READ) Refused }\end{array}$

PN: ROTATE VERBIAGE IN PARENS. ROTATE RESPONSE OPTIONS TO MATCH QUESTION TEXT.

ASK ALL

21. Thinking of the future and your organization's hiring practices, would you say your organization needs to (place greater emphasis on educational degrees or credentials and less on skills and life experience) or the other way around, (place greater emphasis on skills and life experience and less on educational degrees or credentials)?

$(n=650)$

19\% Place greater emphasis on educational degrees or credentials and less on skills and life experience

$66 \%$ Place greater emphasis on skills and life experience and less on educational degrees or credentials

$10 \%$ (DO NOT READ) Neither of these reflect my view

4\% (DO NOT READ) Don't know

$1 \% \quad$ (DO NOT READ) Refused

ASK ALL

(PREVIOUS Q18)

22. With regard to each skill I read, please tell if, in your opinion, workers age 50 and older generally have the advantage over younger workers about age 30 and younger in your organization. [RANDOMIZE AND INSERT a-p; PROMPT AFTER FIRST: Would you say workers age 50 and older are at an advantage over younger workers with this skill or not?

a. Adaptability to change

$(n=650)$

22\% YES, workers age 50 and older are at an advantage over younger workers with this skill [DO NOT READ]

$60 \%$ NO, workers age 50 and older are NOT at an advantage over younger workers with this skill [DO NOT READ] (DO NOT ALLOW OTHER SPECIFY)

$2 \%$ (DO NOT READ) None of these reflect my view (DO NOT ALLOW OTHER SPECIFY)

$13 \%$ (DO NOT READ) Both the same advantage/disadvantage

1\% (DO NOT READ) Don't know (DO NOT ALLOW OTHER SPECIFY)

$1 \%$ (DO NOT READ) Refused (DO NOT ALLOW OTHER SPECIFY) 
b. Basic skills, like math, basic writing, or reading skills

$(n=650)$

39\% YES, workers age 50 and older are at an advantage over younger workers with this skill [DO NOT READ]

$34 \%$ NO, workers age 50 and older are NOT at an advantage over younger workers with this skill [DO NOT READ] (DO NOT ALLOW OTHER SPECIFY)

$4 \%$ (DO NOT READ) None of these reflect my view (DO NOT ALLOW OTHER SPECIFY)

$20 \%$ (DO NOT READ) Both the same advantage/disadvantage

2\% (DO NOT READ) Don't know (DO NOT ALLOW OTHER SPECIFY)

$1 \%$ (DO NOT READ) Refused (DO NOT ALLOW OTHER SPECIFY)

c. Technical skills like engineering, mechanical, machine operation, etc.

$(n=650)$

$30 \%$ YES, workers age 50 and older are at an advantage over younger workers with this skill [DO NOT READ]

43\% NO, workers age 50 and older are NOT at an advantage over younger workers with this skill [DO NOT READ] (DO NOT ALLOW OTHER SPECIFY)

6\% (DO NOT READ) None of these reflect my view (DO NOT ALLOW OTHER SPECIFY)

$15 \%$ (DO NOT READ) Both the same advantage/disadvantage

3\% (DO NOT READ) Don't know (DO NOT ALLOW OTHER SPECIFY)

2\% (DO NOT READ) Refused (DO NOT ALLOW OTHER SPECIFY)

e. Management or leadership skills

$(n=650)$

59\% YES, workers age 50 and older are at an advantage over younger workers with this skill [DO NOT READ]

$22 \% \quad$ NO, workers age 50 and older are NOT at an advantage over younger workers with this skill [DO NOT READ] (DO NOT ALLOW OTHER SPECIFY)

3\% (DO NOT READ) None of these reflect my view (DO NOT ALLOW OTHER SPECIFY)

$14 \%$ (DO NOT READ) Both the same advantage/disadvantage

2\% (DO NOT READ) Don't know (DO NOT ALLOW OTHER SPECIFY)

$1 \%$ (DO NOT READ) Refused (DO NOT ALLOW OTHER SPECIFY) 
f. Team work or collaboration

$(n=650)$

39\% YES, workers age 50 and older are at an advantage over younger workers with this skill [DO NOT READ]

$35 \% \quad$ NO, workers age 50 and older are NOT at an advantage over younger workers with this skill [DO NOT READ] (DO NOT ALLOW OTHER SPECIFY)

$4 \%$ (DO NOT READ) None of these reflect my view (DO NOT ALLOW OTHER SPECIFY)

$18 \%$ (DO NOT READ) Both the same advantage/disadvantage

2\% (DO NOT READ) Don't know (DO NOT ALLOW OTHER SPECIFY)

$1 \%$ (DO NOT READ) Refused (DO NOT ALLOW OTHER SPECIFY)

g. Information and digital technology

$(n=650)$

10\% YES, workers age 50 and older are at an advantage over younger workers with this skill [DO NOT READ]

$75 \% \quad N O$, workers age 50 and older are NOT at an advantage over younger workers with this skill [DO NOT READ] (DO NOT ALLOW OTHER SPECIFY)

$3 \%$ (DO NOT READ) None of these reflect my view (DO NOT ALLOW OTHER SPECIFY)

$10 \%$ (DO NOT READ) Both the same advantage/disadvantage

$1 \%$ (DO NOT READ) Don't know (DO NOT ALLOW OTHER SPECIFY)

1\% (DO NOT READ) Refused (DO NOT ALLOW OTHER SPECIFY)

h. Analytical skills like collecting and analyzing data for decision making

$(n=650)$

34\% YES, workers age 50 and older are at an advantage over younger workers with this skill [DO NOT READ]

$41 \% \quad$ NO, workers age 50 and older are NOT at an advantage over younger workers with this skill [DO NOT READ] (DO NOT ALLOW OTHER SPECIFY)

$4 \%$ (DO NOT READ) None of these reflect my view (DO NOT ALLOW OTHER SPECIFY)

$17 \%$ (DO NOT READ) Both the same advantage/disadvantage

3\% (DO NOT READ) Don't know (DO NOT ALLOW OTHER SPECIFY)

$2 \% \quad$ (DO NOT READ) Refused (DO NOT ALLOW OTHER SPECIFY)

i. Fluency in another language other than English

$(n=650)$

14\% YES, workers age 50 and older are at an advantage over younger workers with this skill [DO NOT READ]

59\% NO, workers age 50 and older are NOT at an advantage over younger workers with this skill [DO NOT READ] (DO NOT ALLOW OTHER SPECIFY)

$6 \%$ (DO NOT READ) None of these reflect my view (DO NOT ALLOW OTHER SPECIFY)

$14 \%$ (DO NOT READ) Both the same advantage/disadvantage

5\% (DO NOT READ) Don't know (DO NOT ALLOW OTHER SPECIFY)

$2 \%$ (DO NOT READ) Refused (DO NOT ALLOW OTHER SPECIFY) 
j. Customer service skills

$(n=650)$

42\% YES, workers age 50 and older are at an advantage over younger workers with this skill [DO NOT READ]

$32 \% \quad$ NO, workers age 50 and older are NOT at an advantage over younger workers with this skill [DO NOT READ] (DO NOT ALLOW OTHER SPECIFY)

$4 \%$ (DO NOT READ) None of these reflect my view (DO NOT ALLOW OTHER SPECIFY)

$18 \%$ (DO NOT READ) Both the same advantage/disadvantage

2\% (DO NOT READ) Don't know (DO NOT ALLOW OTHER SPECIFY)

$1 \%$ (DO NOT READ) Refused (DO NOT ALLOW OTHER SPECIFY)

k. Creativity and innovation

$(n=650)$

14\% YES, workers age 50 and older are at an advantage over younger workers with this skill [DO NOT READ]

$63 \%$ NO, workers age 50 and older are NOT at an advantage over younger workers with this skill [DO NOT READ] (DO NOT ALLOW OTHER SPECIFY)

3\% (DO NOT READ) None of these reflect my view (DO NOT ALLOW OTHER SPECIFY)

$16 \%$ (DO NOT READ) Both the same advantage/disadvantage

3\% (DO NOT READ) Don't know (DO NOT ALLOW OTHER SPECIFY)

$1 \%$ (DO NOT READ) Refused (DO NOT ALLOW OTHER SPECIFY)

I. Problem solving skills

$(n=650)$

52\% YES, workers age 50 and older are at an advantage over younger workers with this skill [DO NOT READ]

$25 \%$ NO, workers age 50 and older are NOT at an advantage over younger workers with this skill [DO NOT READ] (DO NOT ALLOW OTHER SPECIFY)

$4 \%$ (DO NOT READ) None of these reflect my view (DO NOT ALLOW OTHER SPECIFY)

$16 \%$ (DO NOT READ) Both the same advantage/disadvantage

2\% (DO NOT READ) Don't know (DO NOT ALLOW OTHER SPECIFY)

$1 \%$ (DO NOT READ) Refused (DO NOT ALLOW OTHER SPECIFY)

m. Mentoring, coaching, training skills

$(n=650)$

63\% YES, workers age 50 and older are at an advantage over younger workers with this skill [DO NOT READ]

20\% NO, workers age 50 and older are NOT at an advantage over younger workers with this skill [DO NOT READ] (DO NOT ALLOW OTHER SPECIFY)

$3 \%$ (DO NOT READ) None of these reflect my view (DO NOT ALLOW OTHER SPECIFY)

$11 \%$ (DO NOT READ) Both the same advantage/disadvantage

$1 \%$ (DO NOT READ) Don't know (DO NOT ALLOW OTHER SPECIFY)

2\% (DO NOT READ) Refused (DONOTAL OW OTHFR SPFCIEY) 
n. Artistic or design skills

$(n=650)$

12\% YES, workers age 50 and older are at an advantage over younger workers with this skill [DO NOT READ]

$65 \%$ NO, workers age 50 and older are NOT at an advantage over younger workers with this skill [DO NOT READ] (DO NOT ALLOW OTHER SPECIFY)

5\% (DO NOT READ) None of these reflect my view (DO NOT ALLOW OTHER SPECIFY)

13\% (DO NOT READ) Both the same advantage/disadvantage

3\% (DO NOT READ) Don't know (DO NOT ALLOW OTHER SPECIFY)

$2 \%$ (DO NOT READ) Refused (DO NOT ALLOW OTHER SPECIFY)

o. Work ethic and professionalism

$(n=650)$

63\% YES, workers age 50 and older are at an advantage over younger workers with this skill [DO NOT READ]

$18 \%$ NO, workers age 50 and older are NOT at an advantage over younger workers with this skill [DO NOT READ] (DO NOT ALLOW OTHER SPECIFY)

3\% (DO NOT READ) None of these reflect my view (DO NOT ALLOW OTHER SPECIFY)

$12 \%$ (DO NOT READ) Both the same advantage/disadvantage

2\% (DO NOT READ) Don't know (DO NOT ALLOW OTHER SPECIFY)

$1 \%$ (DO NOT READ) Refused (DO NOT ALLOW OTHER SPECIFY)

p. Presentation and public speaking skills

$(n=650)$

37\% YES, workers age 50 and older are at an advantage over younger workers with this skill [DO NOT READ]

$38 \%$ NO, workers age 50 and older are NOT at an advantage over younger workers with this skill [DO NOT READ] (DO NOT ALLOW OTHER SPECIFY)

$4 \%$ (DO NOT READ) None of these reflect my view (DO NOT ALLOW OTHER SPECIFY)

$16 \%$ (DO NOT READ) Both the same advantage/disadvantage

2\% (DO NOT READ) Don't know (DO NOT ALLOW OTHER SPECIFY)

2\% (DO NOT READ) Refused (DO NOT ALLOW OTHER SPECIFY) 
q. Other: (specify) [IF PROVIDED - PROMPT/ASK: DO WORKERS 50+ HAVE AN ADVANTAGE OVER YOUNGER WORKERS WITH THAT SKILL OR NOT]

$(n=650)$

8\% YES, workers age 50 and older are at an advantage over younger workers with this skill [DO NOT READ]

$38 \% \quad$ NO, workers age 50 and older are NOT at an advantage over younger workers with this skill [DO NOT READ] (DO NOT ALLOW OTHER SPECIFY)

7\% (DO NOT READ) None of these reflect my view (DO NOT ALLOW OTHER SPECIFY)

$11 \%$ (DO NOT READ) Both the same advantage/disadvantage

26\% (DO NOT READ) Don't know (DO NOT ALLOW OTHER SPECIFY)

$10 \%$ (DO NOT READ) Refused (DO NOT ALLOW OTHER SPECIFY)

\section{Firmographics}

\section{PN: Insert time stamp.}

SHOW ALL

These last few questions are for classification purposes only and will help us to better understand your answers. Neither your name nor the name of your company will be associated with any of your responses.

\section{ASK ALL}

Z1. Which of these best describes your business? Are you ...? [READ LIST. ENTER ONE ONLY]

$(n=650)$

13\% Publicly owned (your company has shares of stock that the public can buy and sell on the stock market)

61\% Privately owned

$17 \% \quad$ Non-profit, or

$6 \% \quad$ Something else (SPECIFY)

1\% (DO NOT READ) Don't know

$2 \% \quad$ (DO NOT READ) Refused

ASK ALL

Z2. What proportion of the employees at your organization are age 50 or older? Your best guess is fine.

$(n=650)$

$4 \% \quad$ Less than $10 \%$

$17 \% \quad 10$ to less than $25 \%$

$49 \% \quad 25 \%$ to less than $50 \%$

$22 \% \quad 50 \%$ to less than $75 \%$

$2 \% \quad 75 \%$ or more

$4 \%$ (DO NOT READ) Don't know

2\% (DO NOT READ) Refused 
SHOW ALL

Z6. [Record gender by observation]

$\begin{array}{ll}\text { ( } n=650) & \\ & \\ \text { (DO NOT READ) } \\ 23 \% & \text { Male } \\ 77 \% & \text { Female } \\ <1 \% & \text { Unsure }\end{array}$

ASK ALL

Z7. Thank you for your responses in our survey! In order to send you the check for [\$20] as our way of saying thank you for participating in the study, we will need your full name and mailing address. This information will only be used to mail you your [\$20] check. Would you like to provide this information?

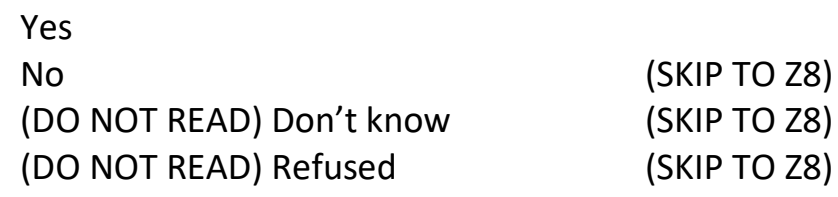

ASK THOSE WILLING TO PROVIDE NAME AND ADDRESS ( $z 7=1)$.

Z7a. [Enter name and address. Verify spelling of name and address before proceeding.]

Full Name:

Company (Optional):

Address:

City:

State:

ZIP:

$P N$ : Ask $Z 8$ if $Z 7=2,8$, or 9. Otherwise, skip to $Z 9$.

ASK THOSE WHO DON'T WANT TO GIVE PERSONAL INFORMATION

Z8. Would you prefer to donate your $\$ 20$ to the American Red Cross instead?

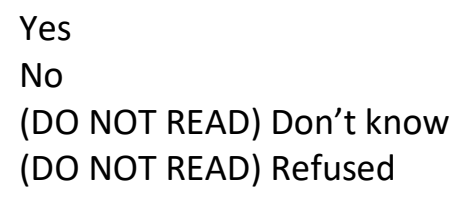

PN: If information provided in $\mathrm{Z7a}$, do not present. If no information provided in $\mathrm{Z7a}$, present second wording. In either case, present phone number.

ASK ALL 
Z9. (If name provided in Z7a) Finally, for verification purposes, did I reach you by dialing:

VERIFY PHONE NUMBER:

(If name not provided) Finally, for verification purposes, may I please have your first name:

VERIFY NAME:

And did I reach you by dialing:

VERIFY PHONE NUMBER:

\section{(CLOSING)}

SHOW ALL

Those are all the questions I have. Thank you very much for your time and opinions.

PN: Insert end time stamp.

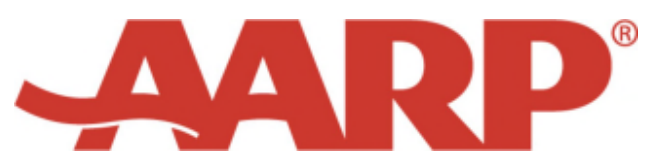

For more information on this issue, please contact

Heather Tinsley-Fix, Senior Advisor, Employer Engagement at htinsley-fix@aarp.org

For more information about this survey or the methodology, please contact Jennifer Sauer, Sr. Research Advisor at jsauer@aarp.org 